\title{
PREFACE: ASSESSMENT OF DAMAGE PROGRESSION MODELS FOR SiC/SiC CERAMIC MATRIX COMPOSITES
}

\section{INTRODUCTION}

The objective of the Assessment of Damage Progression Models for SiC/SiC ceramic matrix composites (CMCs) effort was to assess and quantify the effectiveness of progressive damage models (PDM) for prognosis of behavior and life of $\mathrm{SiC} / \mathrm{SiC}$ ceramic matrix composites in application-relevant environments. This effort attempted to quantify the strengths and limitations associated with the use of current modeling capabilities to predict progressive damage in CMC structures. The work performed under this program are described in eight separate papers in this special edition of the journal, and a ninth paper describes the validation test results.

\section{BACKGROUND}

CMCs are high-temperature materials with one-third the density of metals. They were initially developed in the mid1980s and first flown commercially by GE Aviation (GEA) in August 2016. Specifically, the first CMC commercial components were aerospace $\mathrm{SiC}$ fiber-reinforced $\mathrm{SiC}$ matrix turbine engine hot section shrouds in the Leading Edge Aviation Propulsion (LEAP) engine powering an Airbus A320neo (Steibel, 2019). These SiC/SiC CMC shrouds have performed well since their insertion, and they are now routinely flying in commercial aerospace turbine engines (GEA LEAP) that power both the Airbus A320neo and the Boeing 737 MAX. As of 2021, GEA has shipped over $10 \times 10^{4}$ shrouds.

There are several reasons why it took $\sim 35$ years for CMCs to reach commercial service from the time that they first received significant attention by a plethora of research labs around the world (Steibel, 2019; Padture, 2016; Zok, 2016). When CMCs were first being developed, the cost of the fibers was very high. Additionally, the earlier CMC variants exhibited poor high-temperature durability and stability. Subsequent advances were made in fibers, fiber coatings, and the development of more advanced matrices. These improvements have resulted in CMCs displacing $\mathrm{Ni}$-based superalloy turbine engine components. The insertion of CMCs into turbine engines required designers to leverage their exceptional high-temperature capability while avoiding their known damage modes and limited strain capability. The key to this success was the need to address the flight safety requirements inherent to all aerospace turbine engine hot section components.

When GEA developed the LEAP engine with $\mathrm{SiC} / \mathrm{SiC} \mathrm{CMC}$ shrouds, they made the decision to incorporate these new CMC components based on a safe life approach (Suresh, 1998; Grandt, 2004; Larsen, 2010). The approach employed to introduce the GEA shrouds started with the identification of the key turbine engine operational cycle points that generate the most critical combined stress and temperature points for the shroud. The laminate stresses at these critical cycle points were then compared against well over 100 material response curves generated from mechanical behavior test data that included the measured response for tensile, stress versus cycles, creep strain versus time, as well as unique dwell fatigue with precision strain control types of loading (Steibel, 2019). In this manner, the component life could be empirically estimated using this large mechanical test database. Although this approach proved effective for safe life determination and certification of these new CMC-based turbine engine components, the mechanical database necessary to implement this approach required over $10 \times 10^{5} \mathrm{~h}$ of test time and tens of thousands of test hours for features. Generating this enormous data set cost tens of millions of dollars. Additionally, the database took several years to generate (Steibel, 2021).

Generally, it is well understood that a safe life approach results in conservative operational stress levels. In addition, any significant material changes require the generation of a new multi-million dollar database. Therefore, continued development of new CMC variants for use in critical applications with stringent certification requirements are 
cost-prohibitive, even though these applications are where these materials can provide the greatest benefit. Damagetolerant design approaches (Suresh, 1998; Grandt, 2004) have been employed successfully for years in life-critical metallic components and offer significant advantages over the more conservative safe life approach. Specifically, the damage-tolerant design approach is based on a physics-based probability of detection (PDA) of a critical flaw size and the time it would take that flaw to produce a catastrophic damage state in the material. The requirement to produce such a large data base each time there is a significant material change is daunting; hence, the entire CMC community clearly recognizes that a transition to a more physics-based lifing strategy, such as the damage-tolerant approach, is crucially needed.

Over the last ten years, there has been an increased effort in the CMC aerospace community to transition to a damage tolerance approach. In 2011, the U.S. Air Force Research Laboratory (AFRL) presented a workshop on CMC lifing technology that identified the importance of taking an Integrated Computational Materials Science and Engineering (ICMSE) approach to CMC development and component design along with the need to develop information-rich experiments for the verification and validation of material models. After the workshop, AFRL issued four CMC behavior and lifing contracts to advance the development of analysis tools necessary to transition from a curve fitting empirical lifing approach to a damage tolerance approach for CMC safety critical parts.

Over the past five years, the scientific community has made significant strides in understanding the behavior of CMCs, including complex damage mechanisms, oxidation kinetics, the role of fiber coatings, fatigue and creep mechanisms, and the role of microstructure, and defects. Several of these analysis methods still rely on a calibration phase that requires test data on specimens with the specific fiber architecture of interest. One major limitation is that these models cannot be generalized to make predictions for different laminate stacking sequences or off-axis ply layups, without recalibration of those models using test data with those specific layups.

It has been a significant challenge for the CMC analysis community to effectively predict time-dependent deformation. For CMCs operating at high temperatures $\left(\sim 1500-2400^{\circ} \mathrm{F}\right)$, it is critical that PDA-based methods be closely coupled with analysis methods that account for oxidation kinetics. In addition, the analysis tools need to be microstructurally sensitive, accurately represent the statistical nature of the CMC microstructure, include identification of key microstructural features, and model each individual ply.

Beyond smooth bar test geometries, PDA-based methods need to accurately quantify durability in the presence of stress concentrating geometric features. Life prognosis and damage tolerance in the presence of stress concentrations requires an understanding of the relationship between observed mechanical behavior in smooth bar test specimens and sub-element test specimens with stress concentrations. Stress concentrations will result in a localized shear component that introduces a significantly different matrix cracking pattern than what is observed in (0/90) smooth bars pulled in uniaxial tension. Applying standard linear elastic analysis to component geometries results in an overly conservative and pessimistic expectation of durability for these materials. Furthermore, test specimens with stress concentrations do not demonstrate life-limiting behavior when local stresses in the stress concentration region reach levels associated with the proportional limit observed in smooth bar test specimens. Obviously, there is a lack of understanding of the relationship of progressive damage in smooth bar specimens and progressive damage development in specimens that have a stress concentration. Damage zones are highly susceptible to oxidation, which can lead to an increase in damage propagation rates depending on the loading type and the environment.

For PDA-based methods to work, these models must be physics-based and be able to account for coupling of damage mechanisms (matrix cracking and oxidation) as described earlier. They must address stress concentrations that will be present as the result of geometric shapes of turbine engine components, accurately represent the CMC microstructure and unique features, and eventually be integrated into an overall ICMSE platform.

The U.S. AFRL recently completed the Enhanced Physics-based Prognosis and Inspection of CMC (EPPIC) program, a benchmarking program to assess the current state-of-the-art (SOA) in PDA tools for high-temperature CMCs. The technical objective of this program was to assess the current predictive capabilities of the leading PDA tools and methods for CMCs. The ultimate goal is to be able to transition from a safe life-design approach to a damage-tolerance assessment approach.

A total of eight analysis teams were assembled for the EPPIC program. The teams and their PDM tools were as follows: AlphaStar's GENOA, Columbia's Multi-Scale Design, ATA-Engineering's Binary Model, Teledyne's Bi- 
nary Model, Raytheon's Commercial CLIP Model, Structural Analytics' Clip Model, UDRI's BSAM Model coupled with UES Environmental Model, and GE Aviation's MAC/GMC + CZ. Each of the analysis teams performed blind predictions for up to seven different uniquely loaded feature tests, which were identified as challenge problems. The challenge problems were specifically selected to thoroughly test each model's predictive capabilities in being able to accurately describe the development of damage, retained properties, and life. The challenge problems involved tension, creep rupture, notched fatigue, open-hole creep, and notched four-point flexure fatigue tests and were designed to be sufficiently difficult to push the modeling teams out of their comfort zone. In parallel to the modeling work, validation tests for each challenge problem were conducted. Each modeling analysis team was required to submit their blind predictions before being allowed to review the results of the validation tests. They were then allowed to go back and make changes to their tools and redo their analysis if time and resources allowed.

\section{CERAMIC MATRIX COMPOSITE MATERIALS INVESTIGATED}

The AFRL EPPIC program considered the efficacy of the participating damage models for both a prepreg and a woven $\mathrm{SiC} / \mathrm{SiC} \mathrm{CMC}$ system. The prepreg system was supplied by GE Aviation. This CMC system consists of prepreg tape Silicon Melt Infiltrated Hi-Nicalon ${ }^{\mathrm{TM}}-\mathrm{S} / \mathrm{BN} / \mathrm{Si}_{3} \mathrm{~N}_{4} / \mathrm{SiC}$. This $\mathrm{CMC}$ will be referred to as the GEA SiC/SiC CMC. A unique symmetric unbalanced layup of $[0 / 30 / 60 / 90]_{s}$ was selected for the challenge problems and produced panels that were $\sim 1.6 \mathrm{~mm}$ thick. A schematic of the processing methodology used to make the prepreg tape $\mathrm{SiC} / \mathrm{SiC}$ is shown in Fig. 1 (Steibel, 2019). Silicon-carbide fiber is coated with $\mathrm{BN}$ and then $\mathrm{Si}_{3} \mathrm{~N}_{4}$. The coated fiber is run through a slurry to infiltrate the fiber tow with matrix precursor, and then it is wrapped onto a drum to form the prepreg tape. The tapes are then trimmed and stacked in the desired stacking sequence. The stack-up is then compacted, followed by silicon melt infiltration. The typical microstructure of the $\mathrm{GEA} \mathrm{SiC/SiC}$ is presented in Fig. 2. A tensile specimen was sectioned $90 \mathrm{deg}$ from the loading axis to generate the micrograph. The micrograph documents the excellent density of this CMC system.

The woven system was supplied by COI Ceramics (San Diego, CA, USA) and was the S200H system. This CMC system consists of Hi-Nicalon fibers with a $\mathrm{BN} / \mathrm{Si}_{3} \mathrm{~N}_{4}$ coating in a woven $(8 \mathrm{HSW})$ architecture and a $[0 / 90]_{2 s}$ eight ply layup. The test specimens were $\sim 2.3 \mathrm{~mm}$ thick. A schematic of the processing methodology used to make the woven $\mathrm{SiC} / \mathrm{SiC}$ is shown in Fig. 3. Fabric is first coated with $\mathrm{BN}$ and $\mathrm{Si}_{3} \mathrm{~N}_{4}$. The fibers are then combined with a

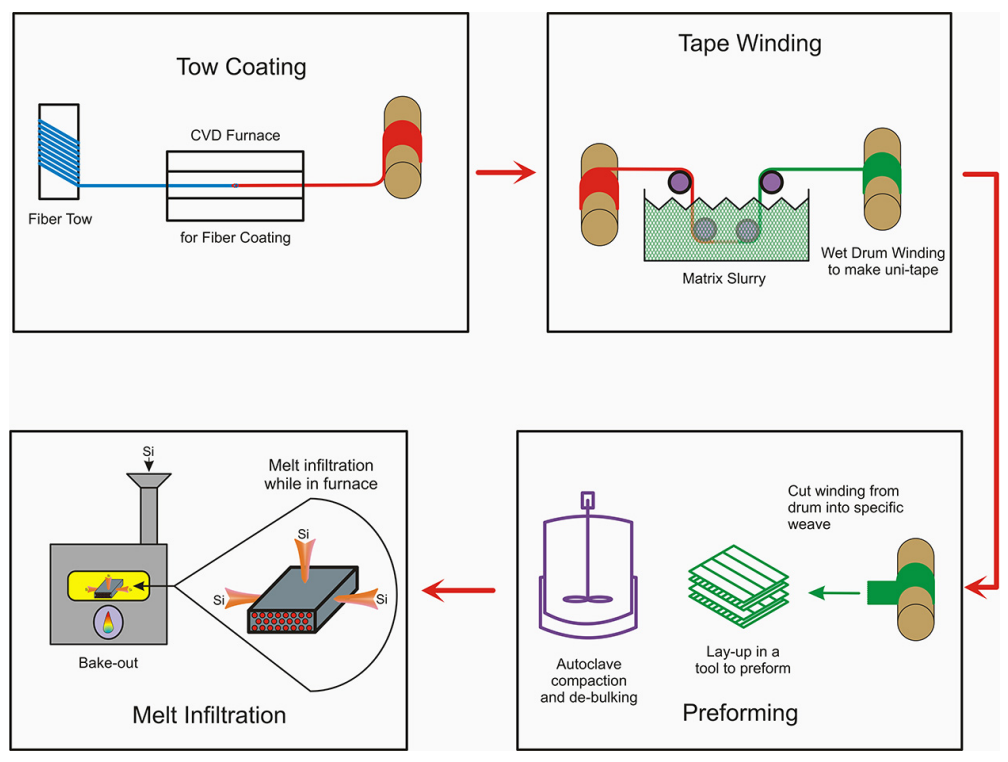

FIG. 1: Schematic of GEA $\mathrm{SiC} / \mathrm{SiC} \mathrm{CMC}$ manufacturing process 


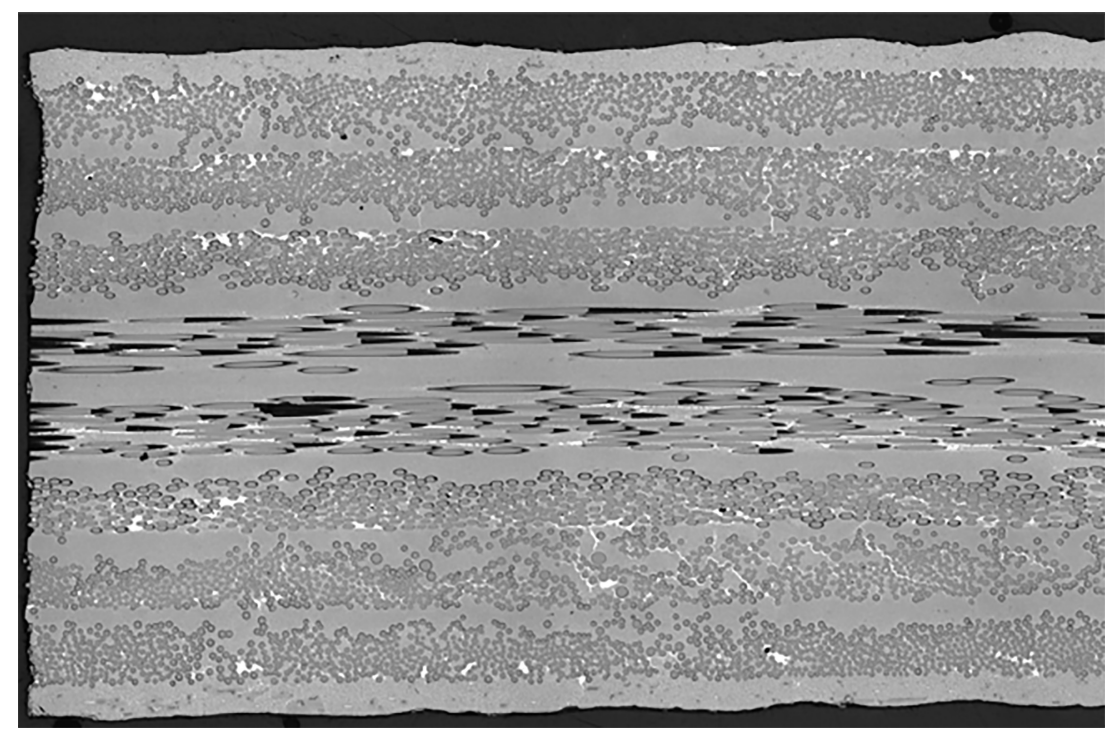

FIG. 2: Optical micrograph of GEA $\mathrm{SiC} / \mathrm{SiC} \mathrm{CMC}$ with a $[0 / 30 / 60 / 90]_{s}$ layup

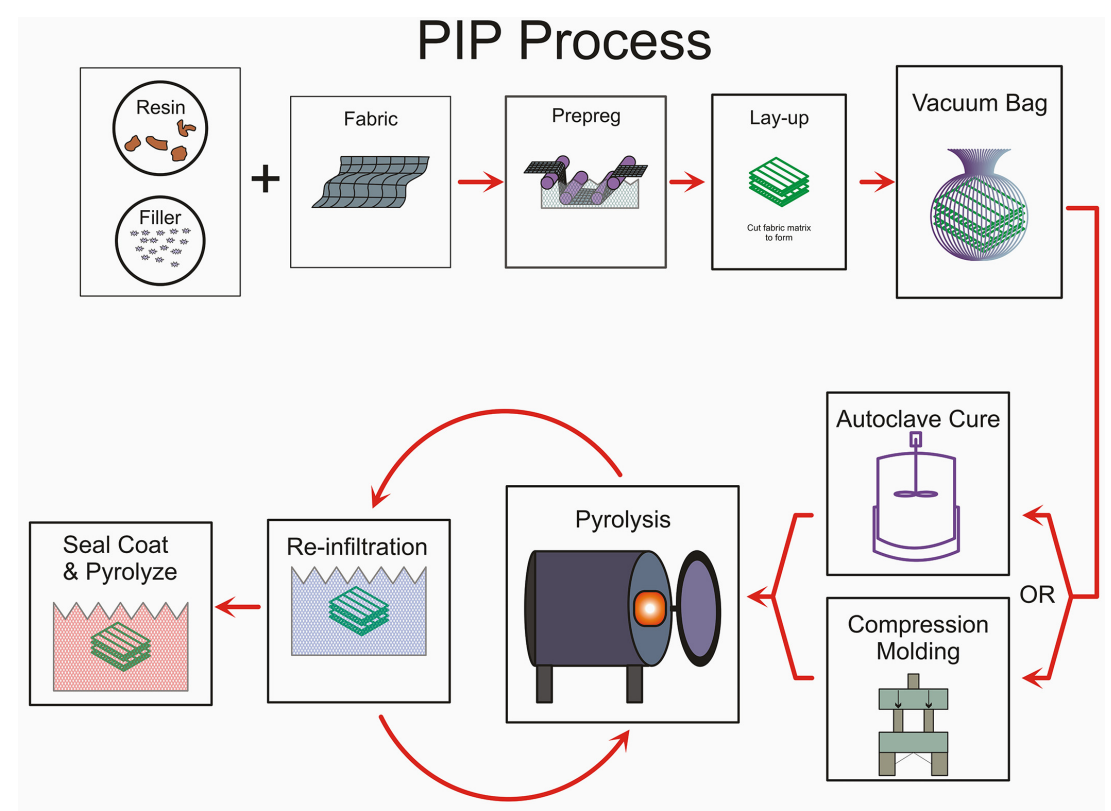

FIG. 3: Schematic of S200H CMC manufacturing process

filler and resin using a prepreg process. The pre-pregged cloth is trimmed and stacked using a set stacking sequence. The stack-up is then autoclave cured followed by machining. The condensed stack-up then pyrolyzed. The infiltration and pyrolysis step is then repeated multiple times until the desired density is achieved. A micrograph showing the typical microstructure of the $\mathrm{S} 200 \mathrm{H} \mathrm{CMC}$ is provided in Fig. 4. A tensile specimen was sectioned 90 deg from the loading axis to generate the micrograph. There is some macro-porosity; however, in general the preform was very well infiltrated and densified. 


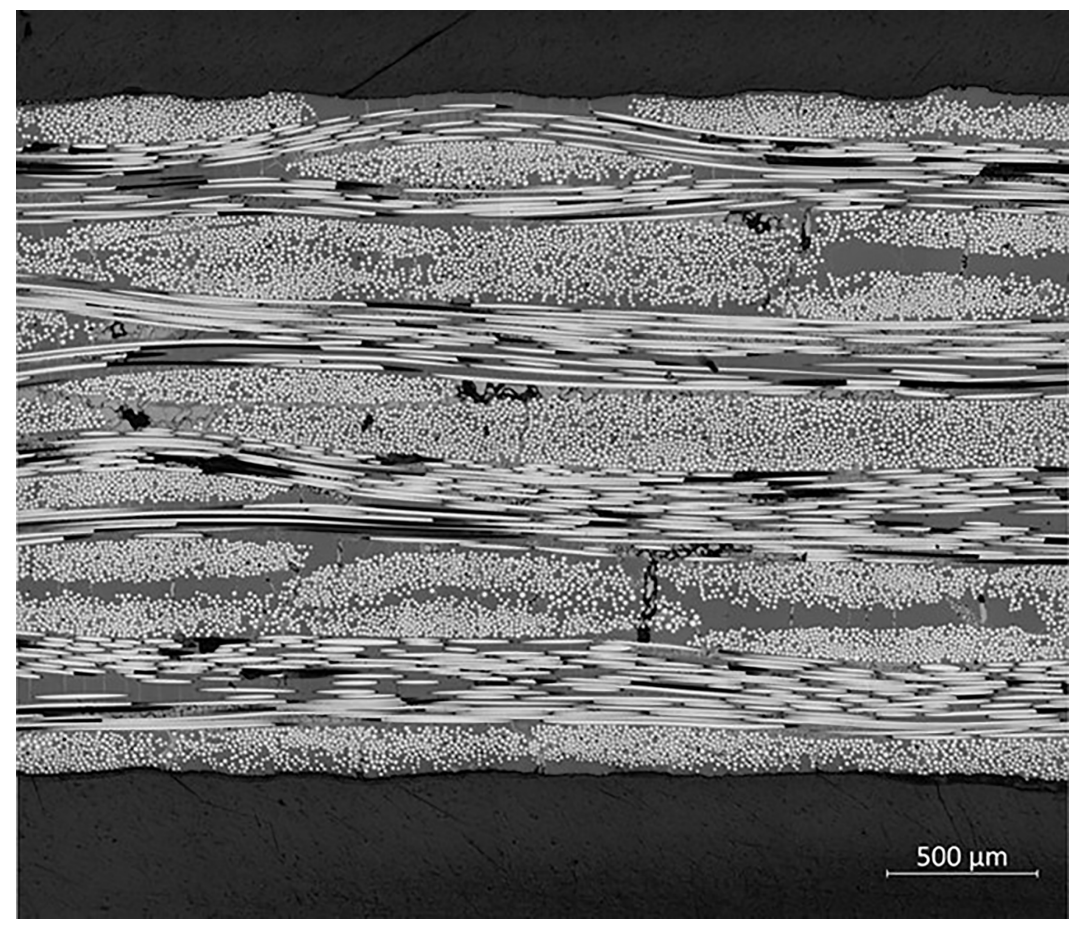

FIG. 4: Optical micrograph of S200H CMC

\section{DESCRIPTION OF CHALLENGE PROBLEMS}

Particular attention went into the selection of the geometries for the actual test specimens for the challenge problems. In general, it was the opinion that the test specimens could either represent features associated with CMC components in aerospace turbine engine hot section components or they could represent sub-elements of actual components. Although arguments could be made for either choice, after significant debate among the team, it was decided to utilize test specimens with features. The reasons for this choice are as follows.

Sub-elements are basically a section out of an actual component. This would typically require the sub-element to be manufactured as a three-dimensional (3D) test specimen. Such a test specimen would typically have both sharp and mild bends, ply drop-offs, curved surfaces, and unique ply layups. Such specimens are often called alphabet test specimens because they take on the shapes of D, C, T, Y, H, V, or I. It is often hard for CMC manufacturers to produce such shapes without defects, especially if sharp corners are required. Manufacturing these shapes often requires several iterations and improvements to the current manufacturing methodology. In addition, selection of a sub-element can require a design that uses a very specific fiber architecture, which the team wanted to avoid. Final test articles often contain significant defects, such as extensive porosity and multiple delaminations. These defects are especially prevalent at the sharp bends or corners. Defect-ridden sub-elements often result in being poor quality interlaminar test specimens and do not provide significant insight into the actual material behavior or capability. As a result, it was decided that such defects would introduce serious risk to the program, and the team did not want to initiate dedicated manufacturing trials just to insure the manufacturability of the required sub-elements.

Testing of sub-elements can also be very challenging. A great deal of time and expense is typically required to design the specialized test fixturing necessary to perform a unique sub-element test and then conduct the test. Additionally, new testing procedures for loading and heating the unique sub-element test specimen need to be developed, potentially introducing variability into the test results. With two CMC systems to be studied, the use of sub-elements was determined not to be a viable option for this program. Use of sub-element test specimens would have introduced increase cost, risk, variability, and most likely resulted in a much longer period of performance for the program. 
Test specimens with features were selected for several strong technical reasons. Flat two-dimensional (2D) panels are ubiquitously employed, especially early on in materials development, and CMC manufacturers can produce flat panels with a high degree of repeatability, and with minimal defects. Delaminations can easily be identified using standard nondestructive investigation techniques. It was determined early on that repeatability was very important to the program, because only a limited number of repeat tests would be conducted for the challenge problems. Appropriate features, such as cooling holes, notches, and dilution holes, are by far the most common features in turbine hot section components, such as airfoils, combustor liners, and shrouds. These features are often associated with damage initiation in CMC components as a result of thermomechanical loading conditions. Each of these features can easily be machined into flat test specimens. Procedures exist for instrumenting flat specimens and testing them using conventional gripping and heating arrangements. Using established testing procedures greatly reduces the chance of experimentally induced scatter in the test results.

The technical team decided to utilize different challenge problems for the two different CMC systems to evaluate a wider range of loading conditions. The loading configurations were selected to be tensile, creep, fatigue, and flexure fatigue. A total of four challenge problems were identified for the GEA SiC/SiC CMC, and three for the S200H $\mathrm{SiC} / \mathrm{SiC} \mathrm{CMC}$. In all cases, the modeling teams were required to submit blind test predictions prior to having access to the test results. After the teams received the test results, they were allowed to adjust their models as required, and perform revised predictions. All the teams were required to report on the strengths and limitations of their approach. The specific test configurations are as follows:

\subsection{Tensile Behavior of GEA SiC/SiC with a Unique Layup}

Teams were required to perform blind predictions of stress versus strain behavior for a tension test specimen of GEA $\mathrm{SiC} / \mathrm{SiC}$ with the unique layup at 23,816 , and $1316^{\circ} \mathrm{C}$. They were to submit blind predictions for tensile stress versus strain behavior and tensile properties of modulus, proportional limit, ultimate tensile strength, and strain to failure. Tensile specimens had the following dimensions: $150 \mathrm{~mm}$ length, $12 \mathrm{~mm}$ width, $28 \mathrm{~mm}$ gage length $=8 \mathrm{~mm}$, gage width, and $317 \mathrm{~mm}$ radius. Tests were conducted under displacement control using a loading rate of $0.04 \mathrm{~mm} / \mathrm{s}$.

\subsection{Tensile Behavior of S200H with Features}

Here the teams were to perform blind predictions of stress versus strain behavior for a tension test specimen of S200H with a unique feature at 23,982 , and $1200^{\circ} \mathrm{C}$. Specifically, the unique features chosen here were two staggered holes that were of similar size to cooling holes used in CMC turbine engine components such as nozzles and shrouds. Each team submitted blind predictions for tensile stress versus edge displacement behavior and tensile properties of ultimate tensile strength and edge displacement to failure. Tensile specimens had the following dimensions: $150 \mathrm{~mm}$ length, $14 \mathrm{~mm}$ width, $28 \mathrm{~mm}$ gage length, $10 \mathrm{~mm}$ gage width, and $317 \mathrm{~mm}$ radius. The holes were $2 \mathrm{~mm}$ in diameter and spaced $3 \mathrm{~mm}$ apart along the width and $3 \mathrm{~mm}$ apart along the length. Each hole was centered $3.5 \mathrm{~mm}$ from the edge of the specimen. Tests were conducted under displacement control using a loading rate of $0.04 \mathrm{~mm} / \mathrm{s}$.

\subsection{Creep Rupture of GEA SiC/SiC}

Each team was asked to perform blind predictions for a creep rupture (CR) test specimen of GEA SiC/SiC with the unique layup at $1316^{\circ} \mathrm{C}$. They were to predict the stress level that resulted in a creep rupture life of $100 \mathrm{~h}$ and predict the tensile stress versus strain behavior and tensile properties for interrupted specimens at $50 \%$ of life. In addition, they were to predict both the strain accumulation versus time and versus life. They also predicted the tensile stress versus strain behavior and tensile properties for the interrupted specimens. Creep rupture specimens had dimensions of: length $=150 \mathrm{~mm}$, width $=12 \mathrm{~mm}$, gage length $=28 \mathrm{~mm}$, gage width $=8 \mathrm{~mm}$, radius $=317 \mathrm{~mm}$. Specimens will be loaded to the creep stress using a rate of $10 \mathrm{MPa} /$ second. 


\subsection{Middle Hole Creep Rupture of S2OOH}

Each team was to perform blind predictions for a middle hole creep rupture (MHCR) test specimen of S200H at $1200^{\circ} \mathrm{C}$. The hole represents a typical dilution hole found in combustors. They each were to predict the stress level that would result in a creep rupture life of $100 \mathrm{~h}$ and predict tensile stress versus edge displacement and ultimate retained tensile strength for interrupted specimens at $50 \%$ of life. Additionally, they were to predict the damage evolution and crack growth and/or damage progression for interrupted specimens and extent of oxidized or damaged zones. MHRC test specimens were $150 \mathrm{~mm}$ long and $24 \mathrm{~mm}$ wide, with an $8 \mathrm{~mm}$ diameter center hole. These specimens were loaded to the creep stress using a rate of $10 \mathrm{MPa} / \mathrm{s}$.

\subsection{Double Edge Notch Fatigue of GEA SiC/SiC}

Each team was to perform blind predictions for a double edge notch fatigue (DENF) test specimen of GEA SiC/SiC tested at $1316^{\circ} \mathrm{C}$ with the unique layup. The notches represent features commonly found in combustors and shrouds. They were to predict stress level that will result in a fatigue life of $50 \mathrm{~h}$ and predict tensile properties for interrupted specimens at $50 \%$ of life. In addition, each team was to predict the damage evolution and crack growth. The DENF test specimens had a length of $150 \mathrm{~mm}$ and a width of $20 \mathrm{~mm}$. The notches were approximately $3 \mathrm{~mm}$ wide and $3 \mathrm{~mm}$ deep, with a notch radius of $1.5 \mathrm{~mm}$. Specimens were fatigue tested at a frequency of $1 \mathrm{~Hz}$ and a load ration of 0.1 .

\subsection{Notched Four-Point Flexural Fatigue of GEA SiC/SiC}

Each team was to perform blind predictions for a notched four-point flexural fatigue (NFPFF) test specimen of GEA $\mathrm{SiC} / \mathrm{SiC}$ tested at $816^{\circ} \mathrm{C}$ for the unique layup and a total of 16 plies. Such a featured specimen does a nice job of representing conditions that are experienced by shrouds. They each were to predict a stress level that would result in a fatigue life of $50 \mathrm{~h}$ and predict flexural strength for interrupted specimens at $50 \%$ of life. Additionally, each team was to predict the damage evolution in terms of matrix cracks and delamination cracks, as well as vertical displacements of the sub-element, and failure load. NFPFF test specimens were $70 \times 8 \mathrm{~mm}$. Tests were conducted at $0.5 \mathrm{~Hz}$ using a load ration (load minimum/load maximum) of $R=0.1$. Material was double the normal thickness and consisted of 16 plies. The layup of the plies was $[0 / 30 / 60 / 90]_{2 s}$. This double thickness resulted in a thickness of $\sim 3.2 \mathrm{~mm}$, and the notch was selected to be approximately three plies deep.

\subsection{Notched Four-Point Flexural Fatigue of S200H}

Each team was to perform blind predictions for a NFPFF test specimen of $\mathrm{S} 200 \mathrm{H}$ tested at $982^{\circ} \mathrm{C}$. Such a featured specimen does a nice job of representing conditions that are experienced by shrouds. They each were to predict the stress level that would result in a fatigue life of $50 \mathrm{~h}$ and predict flexural strength for interrupted specimens at $50 \%$ of life. Tests were conducted at $0.5 \mathrm{~Hz}$ using a load ration (load minimum/load maximum) of $R=0.1$. Each team was to predict the damage evolution in terms of matrix cracks and delamination cracks, as well as vertical displacements of the sub-element, and failure load. Specimens were $75 \times 8 \mathrm{~mm}$ with a notch that was approximately two plies deep.

\section{SUMMARY}

EPPIC was a comprehensive benchmarking program designed to evaluate and advance progressive damage models for CMCs to support a damage tolerance lifing methodology/PDA approach for safety critical CMC components. A total of seven challenge problems were addressed in this study; they are summarized in Table 1. This study determined the initial benefits and problems for using existing PDA capabilities to predict and model damage in CMCs. The program revealed that modeling teams can reasonably predict life for tensile, fatigue, and creep loading conditions if provided with high-quality calibration data. However, they are not able to accurately account for damage progression that occurs during interrupted tests. In addition, much work remains to integrate physics-based oxidative damage mechanisms into the current PDM approaches. 


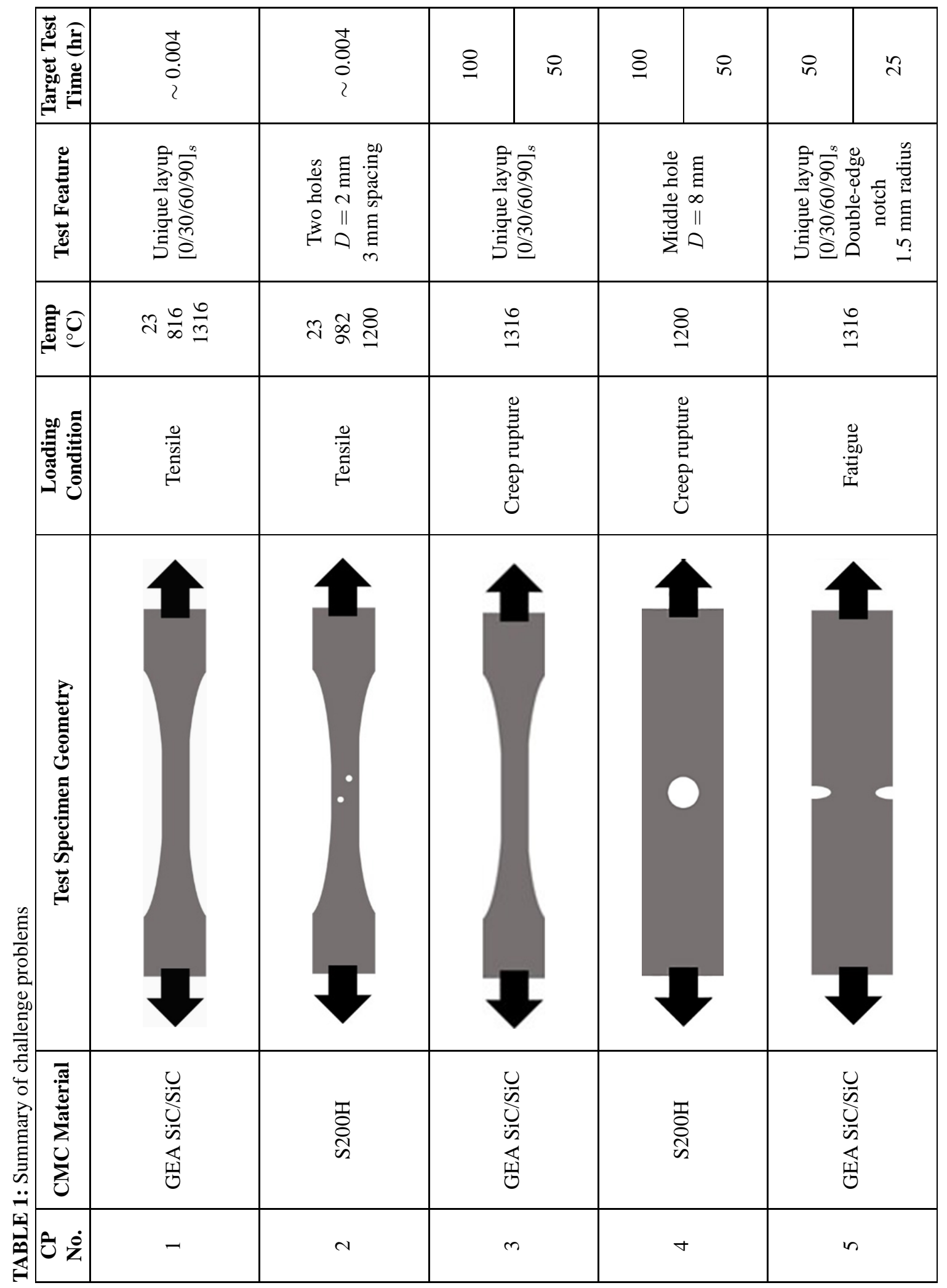




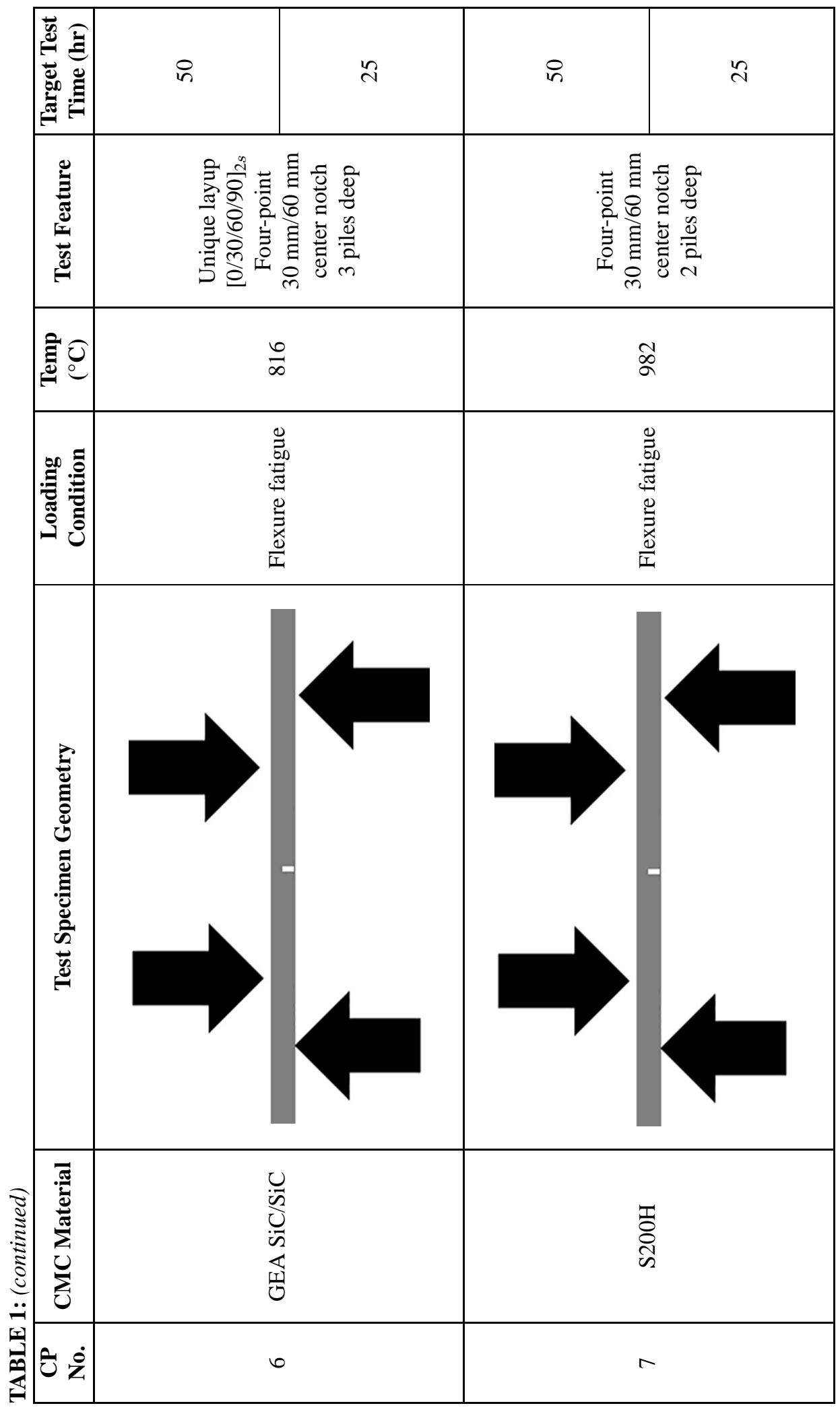


This special issue of the journal contains papers that address the validation test results and presents details of the modeling approaches and blind predictions for all eight modeling teams.

\section{REFERENCES}

Grandt, A.F., Fundamentals of Structural Integrity, Hoboken, NJ: Wiley, 2004.

Larsen, J.M., Understanding Materials Uncertainty for Prognosis of Advanced Turbine Engine Materials, Air Force Tech. Rep. No. AFRL-RX-WP-TP-2010-4139, 2010.

Padture, N.P., Advanced Structural Ceramics in Aerospace Propulsion, Nat. Mater., vol. 15, pp. 804-809, 2016.

Steibel, J., Ceramic Matrix Composites Taking Flight at GE Aviation, Am. Ceram. Soc. Bull., vol. 98, no. 3, pp. 30-33, 2019.

Steibel, J., Ceramic Matrix Composites Taking Flight at GE Aviation, accessed November 5, 2021, from www.youtube.com/Watch?v=9_OaOLS3CWY, 2021.

Suresh, S., Fatigue of Materials, Cambridge, UK: Cambridge University Press, 1998.

Zok, F.W., Ceramic-Matrix Composites Enable Revolutionary Gains in Turbine Engine Efficiency, Am. Ceram. Soc. Bull., vol. 95, no. 5 , pp. 22-28, 2016.

Guest Editors:

George Jefferson

U.S. Air Force, Wright-Patterson AFB

Ohio 45433-7817, USA

Craig Przybyla

U.S. Air Force, Wright-Patterson AFB

Ohio 45433-7817, USA

Larry Zawada

Arctos

Dayton, Ohio 45432-2600, USA 